\title{
Two important aging effects on the martensite phase in CuZnAl alloys. Rubber effect and stabilization of martensite
}

\author{
K. Marukawa and K. Tsuchiya ${ }^{1}$ \\ Department of Applied Physics, Hokkaido University, Sapporo 060-8628, Japan \\ ${ }^{1}$ Department of Production Systems Engineering, Toyohashi University of Technology, \\ Toyohashi 441-8580, Japan
}

Two well-known aging effects on the martensite phase, rubber effect and stabilization of martensite, are closely related to each other. They appear in the same specimen after the same aging treatment. It has been shown that the time constants for these effects are quite similar. However, the causes to bring about these effects may not be the same. For the rubber effect, a short-range-order model has been proposed (K.Marukawa and K.Tsuchiya, Scripta Met. Mater., 32, 77, (1995)). Although somemodifications on the model has been suggested, the original one seems to be most appropriate. In this report, it is shown that the principal cause for the stabilization is the change in the long-range-order of the alloy, while the long-range-order has no relation to the rubber effect. There are two cases for the stabilization in $\mathrm{Cu}-\mathrm{Zn}-\mathrm{Al}$ alloys. In one case, the parent phase has B2 order and the B2 order disintegrates to the disordered state during the aging after the martensitic transformation. In the other case, the matrix phase has L21 structure and the ordered structure transforms to a different ordered structure, DO22. Both these changes in the longrange order result in a stabilization of martensite phase. The relation between these effects and the change in the long-range or short-range order is examined quantitatively with the help of the Monte Carlo simulation. It is shown that the transformation temperature is quite sensitive to the change in the long range order, so that a few percent change is enough to give rise to a change in the transformation temperature of tens of degrees. It is also shown that the changing rates of the long range order and the short range order are comparable with each other in most cases. These results are compared with recent experimental results. 